\title{
Comparação entre dois métodos objetivos e individuais de determinação da velocidade de corrida no limiar de lactato
}

\author{
Fabiana Andrade Machado ${ }^{1}$ \\ Solange Marta Franzói de Moraes ${ }^{2}$ \\ Cecília Segabinazi Peserico ${ }^{3}$ \\ Paulo Victor Mezzaroba ${ }^{3}$ \\ Wonder Passoni Higino 4 \\ ${ }^{1}$ Departamento de Educação Física, Universidade Estadual de Maringá, PR, Brasil \\ 2 Departamento de Ciências Fisiológicas, Universidade Estadual de Maringá, PR, Brasil \\ ${ }_{3}^{3}$ Laboratório de Fisiologia do Esforço, Departamento de Ciências Fisiológicas, \\ Universidade Estadual de Maringá, PR, Brasil \\ ${ }^{4}$ Departamento de Educação Física, UNISALESIANO - \\ Centro Universitário Católico Salesiano Auxilium, Lins, SP, Brasil
}

\begin{abstract}
Resumo: $O$ objetivo deste estudo foi comparar as velocidades de corrida no limiar de lactato (VLL) determinadas pelos métodos objetivos e individuais $\operatorname{Dmax}\left(v L L_{D \max }\right)$ e $\log -\log \left(v L L_{\text {log-log }}\right.$ ) sendo esperado uma relação entre eles melhor do que a relação deles com o método de concentração fixa de lactato sanguíneo em 3,5 mM ( $\left(\mathrm{vL}_{3,5 \mathrm{mM}}\right)$. Participaram deste estudo 17 corredoras recreacionais com experiência em provas de rua de $10 \mathrm{~km}$ (idade: 42,1 $\pm 6,7$ anos; estatura: $1,63 \pm 0,03 \mathrm{~m}$; massa corporal $57,2 \pm 4,9 \mathrm{~kg}$ ) que realizaram um teste incremental em esteira ergométrica, com velocidade inicial de $7 \mathrm{~km} / \mathrm{h}$ e incrementos de $1 \mathrm{~km} / \mathrm{h}$ a cada três minutos. Amostras de sangue foram coletadas nas pausas de 30 segundos entre cada estágio. As vLL foram 10,3 $\pm 1,4$ (vLL $L_{\text {log-log }}$ ), 10,9 $\pm 0,8$ (vLL $\left(\mathrm{vLL}_{3,5 \mathrm{mM}}\right)$ sendo a vLL $\mathrm{L}_{3,5 \mathrm{mM}}$ significantemente superior à $v \mathrm{LL}_{\text {log-log }}(P<0,001)$ e à $v L_{\text {Dmax }}(P=0,024)$. Apesar disso, a amplitude dos limites de concordância ( $\pm 1,96$ DP) entre as vLL foi semelhante, variando entre $\pm 1,48$ e $\pm 1,77 \mathrm{~km} / \mathrm{h}$ e a correlação entre a $v L_{3,5 \mathrm{mM}} \mathrm{e}$ as velocidades $v L L_{\text {log-log }}(r=0,70)$ e vLL $L_{\max }(r=$ $0,78)$ foi maior que a correlação daquelas velocidades entre si $(r=0,65)$. Em suma, as velocidades $v L L_{\text {log-log }}$ e vLL $L_{\text {max }}$ não apresentaram uma melhor relação entre si do que a relação delas com a $v L L_{3,5 \mathrm{mM}}$.
\end{abstract}

Palavras-chave: concordância; comparação; Dmax; log-log.

\section{Comparison between two objective and individual methods of determination of the running speed at lactate threshold}

\begin{abstract}
The aim of this study was to compare the running speed at lactate threshold (sLT) determined by the objective and individual methods $\operatorname{Dmax}\left(s L T_{\text {Dmax }}\right)$ and $\log$-log $\left(s L T_{\log -\log }\right.$ ) being expected a better relationship between them than the relationship of them to the fixed lactate concentration method at $3.5 \mathrm{mM}$ $\left(\mathrm{sLT}_{3.5 \mathrm{mM}}\right)$. Seventeen amateur women runners, with experience of $10 \mathrm{~km}$ road races (age: $42.1 \pm 6.7$ year; height: $1.63 \pm 0.03 \mathrm{~m}$; body mass $57.2 \pm 4.9 \mathrm{~kg}$ ), participated in this study. They performed an incremental exercise test on a motorized treadmill, which the initial speed was $7 \mathrm{~km} / \mathrm{h}$, with increments of $1 \mathrm{~km} / \mathrm{h}$ every 3 min. Blood samples were collected between the stages, during a rest of 30 -s. The sLT were $10.3 \pm 1.4$ (sLT $\left.T_{\text {log-log }}\right), 10.9 \pm 0.8\left(\mathrm{sLT}_{\mathrm{Dmax}}\right)$ and $11.6 \pm 1.2 \mathrm{~km} / \mathrm{h}\left(\mathrm{sLT}_{3.5 \mathrm{~mm}}\right)$ being the $\mathrm{sLT}_{3.5 \mathrm{~mm}}$ significantly higher than $\mathrm{SLT}_{\log -\log }(P<0.001)$ and $\mathrm{SLT}_{\mathrm{Dmax}}(P=0.024)$. Despite that, the limits of agreement range $( \pm 1.96 \mathrm{SD})$ among the SLT was similar, varying between \pm 1.48 and $\pm 1.77 \mathrm{~km} / \mathrm{h}$ and the correlation between $\mathrm{SLT}_{3.5 \mathrm{~mm}}$ and the speeds $\mathrm{sLT}_{\text {log-log }}(r=0.70)$ and $\mathrm{SLT}_{\mathrm{Dmax}}(r=0.78)$ was higher than the correlation between $\mathrm{sLT}_{\text {log-log }}$ and $\operatorname{sLT}_{\operatorname{Dmax}}(r=0.65)$. In summary, the $s L T_{\text {log-log }}$ and $\mathrm{sLT}_{\mathrm{Dmax}}$ speeds did not show a better relationship between themselves than the relationship of them to the $s L T_{3.5 \mathrm{mM} \text {. }}$
\end{abstract}

Keywords: agreement; comparison; Dmax; log-log.

\section{Introdução}

O limiar de lactato (LL) tem sido amplamente utilizado por treinadores, atletas e pesquisadores para a predição do desempenho, prescrição da intensidade de exercício e avaliação dos efeitos do treinamento (BILLAT, 1996; BISHOP et al., 1998; NICHOLSON; SLEIVERT, 2001; PAPADOPOULOS et al., 2006; SJODIN;
JACOBS, 1981; STRATTON et al., 2009). No entanto, muitos métodos foram propostos para a sua detecção, produzindo-se diferentes estimativas deste parâmetro (DAVIS et al., 2007;

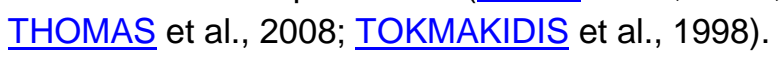

Existem métodos subjetivos, tais como a detecção visual do aumento abrupto na 
concentração de lactato com o aumento da intensidade de exercício (DAVIS et al., 1976) e existem métodos objetivos, tais como métodos baseados na concentração fixa de lactato sanguíneo (KINDERMAN et al., 1979; HECK et al., 1985; SJODIN; JACOBS, 1981) ou métodos baseados em um aumento fixo acima da lactatemia de repouso ( $\underline{\text { COYLE }}$ et al., 1983; FARRELL et al., 1979; YOSHIDA et al., 1987).

A principal desvantagem da detecção visual do LL é a subjetividade implícita neste método, cuja detecção se torna dependente da experiência do pesquisador Davis (2007). De acordo com Beaver (1985), um modelo matemático que se ajuste bem aos dados pode contribuir para a detecção do LL com mais precisão do que técnicas de detecção visual. A desvantagem dos métodos baseados em aumento fixo do lactato acima da concentração de repouso ou basal é que esses métodos não consideram as respostas metabólicas individuais, podendo sub ou superestimar o LL devido às variações inter-individuais na concentração de lactato sanguíneo (DAVIS et al., 2007). Da mesma forma, os métodos de concentração fixa de lactato sanguíneo, como a concentração fixa de 3,5 mM utilizada para se estimar a máxima fase estável em adultos para protocolos com estágios de três minutos (트CK et al., 1985), são limitados por assumirem que essa concentração fixa é válida para todos os sujeitos, sem considerar a variabilidade entre eles (TOKMAKIDIS et al., 1998).

Entre os métodos objetivos que consideram as características metabólicas individuais na detecção do LL incluem-se o método do máximo desvio (Dmax) proposto por Cheng et al. (1992) e o método da transformação logarítmica (log-log) proposto por Beaver et al. (1985). Embora inicialmente sugeridos para a detecção do LL a partir de uma suposta relação curvilinear entre lactato e consumo de oxigênio $\left(\mathrm{VO}_{2}\right)$, estes métodos podem ser aplicados na relação lactatocarga (i.e. potência mecânica), pois também assumem um comportamento curvilinear nesta relação. Por exemplo, o método Dmax identifica o LL na maior distância da curva lactato-carga em um segmento linear interpolado entre o primeiro e último ponto dessa mesma curva. De outro lado, o método log-log transforma os valores brutos da relação lactato-carga para valores em base logarítmica, tornando mais clara a intersecção entre dois segmentos de reta. Um ponto importante é que o método log-log proposto originalmente por Beaver et al. (1985) pode ser considerado subjetivo, pois identifica visualmente o ponto de intersecção. Para ser considerado objetivo, a detecção do ponto de intersecção deve ocorrer matematicamente (MYERS et al., 1994).

Considerando que os métodos log-log e Dmax assumem um comportamento curvilinear na relação lactato-carga, é razoável esperar que eles detectem o LL em intensidades semelhantes. Em adição, seria possível esperar uma forte associação entre estes métodos, principalmente quando comparados a métodos não individualizados de detecção do LL. Deste modo, o objetivo deste estudo foi comparar as velocidades de corrida no limiar de lactato (vLL) determinadas pelos métodos Dmax ( $\left.v L L_{\text {Dmax }}\right)$ e $\log -\log \left(v L_{\text {log-log }}\right)$ examinando a relação entre elas. Adicionalmente, as variáveis $v L_{D \max } \mathrm{e}$ vLL log-log foram comparadas com a vLL determinada através do método de concentração fixa de lactato sanguíneo em 3,5 mM ( $v L_{3,5 \mathrm{~mm}}$ ). Como hipótese, esperávamos que os métodos $L_{\text {Dmax }}$ e a $L_{\text {log-log }}$ provessem a identificação do LL em velocidades coincidentes, com maior concordância quando comparadas com a $\mathrm{vLL}_{3,5 \mathrm{mM} \text {. }}$

\section{Métodos}

\section{Sujeitos}

Dezessete corredoras recreacionais que integravam grupos de corrida da cidade, sem restrição de idade, com experiência em provas de rua de $10 \mathrm{~km}$ aceitaram fazer parte deste estudo. As características antropométricas das participantes foram: idade 42,1 $\pm 6,7$ anos; estatura 1,63 \pm 0,03 $\mathrm{m}$; massa corporal 57,2 \pm 4,9 $\mathrm{kg}$; e índice de massa corporal (IMC) 21,5 \pm 1,8 $\mathrm{kg} / \mathrm{m}^{2}$. As participantes possuíam experiência de $3,1 \pm 1,9$ anos de treinamento com frequência de 2,6 \pm 0,5 sessões de treinamento por semana percorrendo uma distância semanal de 25,9 \pm 6,0 $\mathrm{km}$. Não foi feito nenhum controle sobre as variáveis relacionadas ao climatério. Todas as participantes leram e assinaram o termo de consentimento livre e esclarecido antes do início dos testes. O protocolo utilizado neste estudo foi previamente aprovado pelo Comitê de Ética local (\#719/2010).

\section{Teste incremental em esteira rolante}

$O$ teste incremental foi realizado em uma esteira ergométrica (INBRASPORT Super ATL, Porto Alegre, Brasil). A velocidade inicial foi de 7 
$\mathrm{km} / \mathrm{h}$ com incrementos de $1 \mathrm{~km} / \mathrm{h}$ a cada 3 minutos após um prévio aquecimento de 5 minutos a $5 \mathrm{~km} / \mathrm{h}$. A inclinação da esteira foi mantida em $1 \%$ durante todo o teste. Entre os estágios houve uma pausa de 30 segundos para a coleta de $25 \mu \mathrm{L}$ de sangue do lóbulo da orelha para análise da concentração de lactato sanguíneo através de um analisador eletroquímico (YSI 1500, Ohio, EUA). A escala 620 de Borg (BORG, 1982) foi utilizada para avaliar a percepção subjetiva de esforço (PSE) durante o teste. $O$ teste foi mantido até a exaustão voluntária, sendo que cada participante foi encorajada verbalmente a se manter em exercício pelo maior tempo possível. Cada teste foi considerado válido se pelo menos dois dos seguintes critérios fossem atendidos: 1) frequência cardíaca máxima maior que $90 \%$ da predita pela idade (220-idade); 2) concentração de lactato sanguíneo ao final do teste ([LA]pico) acima de $8 \mathrm{mM}$; 3) PSE ao final do teste (PSEpico) maior que 18 na escala 6-20 de Borg; 4) sinais de intenso esforço (isto é, hiperpnéia, rubor facial, descompasso de passadas).

\section{Determinação do limiar de lactato}

Para a determinação do LL foram utilizados dois métodos com as mesmas características de serem objetivos e individualizados. $O$ primeiro método foi o método Dmax proposto por Cheng et al. (1992). O segundo método foi o método log-log proposto por Beaver et al. (1985). Além disso, o LL foi determinado por meio de interpolação linear na concentração fixa de $3,5 \mathrm{mM}\left(\mathrm{vLL}_{3,5 \mathrm{~mm}}\right)$ ( $\underline{\mathrm{HECK}}$ et al., 1985) para efeitos de comparação com os métodos individualizados.

O método Dmax consiste na determinação do ponto em uma curva ajustada de lactato sanguíneo versus intensidade de exercício, em que ocorre a maior distância perpendicular da linha traçada conectando o primeiro e o último ponto desta curva, conforme indicados na Figura 1. Para tanto, inicialmente os dados (lactato sanguíneo versus velocidade) foram ajustados para a curva exponencial mais constante (Hughson et al., 1987) abaixo representada:

$$
[\mathrm{LA}]=a+b \cdot e^{(c \cdot v)}
$$

em que, $e$ é o número neperiano, $v$ é a velocidade em $\mathrm{km} / \mathrm{h} ; a, b$ e $c$ são os parâmetros da curva; e [LA] é o valor predito da concentração de lactato. Os parâmetros $a, b$ e $c$ foram determinados com o auxílio do Statistical Package for the Social Sciences (SPSS Inc. USA), versão 17.0 .

A função exponencial mais constante foi utilizada visto que conforme Hughson et al. (1987) o aumento da concentração de lactato sanguíneo durante exercício progressivo é bem ajustado por esta função. Além disso, Hughson et al. (1987) demonstraram que esta função, composta pela função exponencial " $b \cdot e^{(c \cdot v) \text { " adicionada à função }}$ constante "a" se ajusta muito melhor aos dados do que a função exponencial simples " $b \cdot e^{(c \cdot v) "}$.

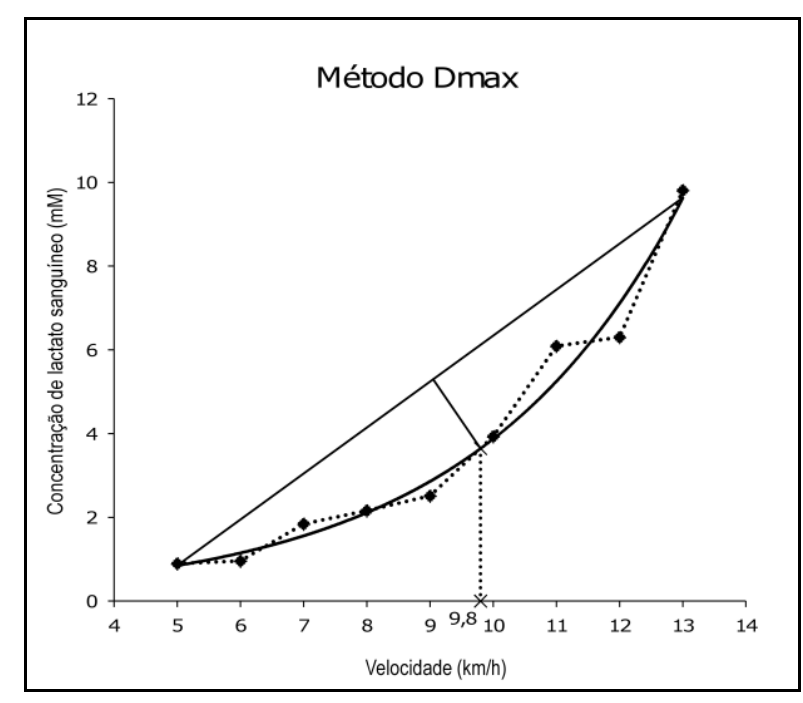

Figura 1. Velocidade no limiar de lactato determinada pelo método Dmax (dados fictícios). Neste exemplo a vLL $L_{\text {max }}$ é $9,8 \mathrm{~km} / \mathrm{h}$.

A máxima distância perpendicular que representa a $v L L_{D \max }$ ocorre no ponto em que a tangente da curva exponencial mais constante é igual ao coeficiente angular da reta traçada conectando o primeiro e o último ponto desta curva. Considerando-se que a tangente de uma curva é obtida através da primeira derivada de sua função, após alguns procedimentos matemáticos, a $v L L_{D \max }$ resultou na seguinte equação:

$$
\begin{gathered}
\operatorname{vLL} L_{\text {max }}=\left(\operatorname { l n } \left(\left(e^{(\text {c.vf })}-e^{(\text {c.vi })}\right) /(c . v f-\right.\right. \\
\text { c.vi }))) / c
\end{gathered}
$$

em que, In é o logaritmo neperiano, e é o número neperiano, $c$ é o parâmetro da curva exponencial mais constante, vi e vf são respectivamente as velocidades inicial e final do teste incremental.

Em relação ao método log-log, inicialmente o logaritmo base 10 da concentração de lactato sanguíneo $\left(\log _{[\llcorner A]}\right)$ e da velocidade $\left(\log _{\mathrm{v}}\right)$ foram calculados. Feito isto, a curva log-log foi traçada e dividida em dois segmentos unidos por um ponto 
em comum inicialmente desconhecido. Uma reta foi ajustada para cada segmento através da regressão linear e a soma dos quadrados dos resíduos (SQR) das duas retas foram adicionados. Outro ponto em comum foi escolhido e o processo foi repetido ao longo de todos os possíveis pontos em comum. O ponto em comum em que a soma dos SRQ foi minimizada foi considerado o ponto de divisão das duas retas (MYERS et al., 1994). Esta rotina foi realizada automaticamente com o auxílio do Microsoft Office Excel 2007. Este procedimento é objetivo e diferente do adotado por Beaver et al. (1985), em que o ponto de divisão foi determinado visualmente.

Após a obtenção objetiva do ponto de divisão, as duas equações lineares (pré e pós este ponto), foram definidas como se segue:

$$
\begin{aligned}
& \log _{[L A] \text { pré }}=a_{\text {pré }}+b_{\text {pré }} \log _{v} \\
& \log _{[L A] \text { pós }}=a_{\text {pós }}+b_{\text {pós }} \log _{v}
\end{aligned}
$$

Calculando-se a intersecção destas duas equações, determina-se o valor $\log _{v}$ como se segue:

$$
\log _{v}=\left(a_{\text {pré }}-a_{\text {pós }}\right) /\left(b_{\text {pós }}-b_{\text {pré}}\right)
$$

Assim, a vLL log-log é igual ao antilogaritmo de $\log _{v}($ Figura 2).

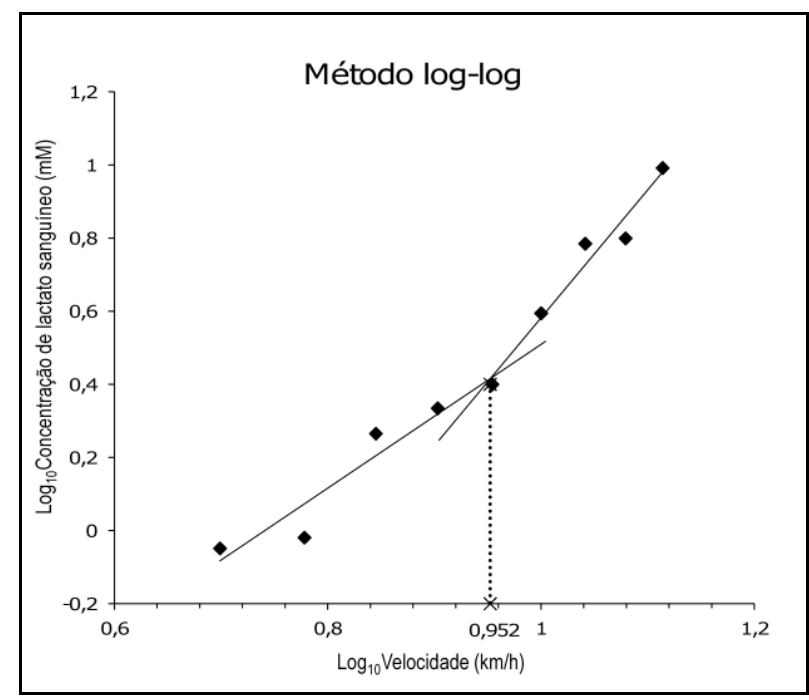

Figura 2. Velocidade no limiar de lactato determinada pelo método log-log ( $\left.\mathrm{vL}_{\mathrm{log}} \mathrm{log}\right)$. Neste exemplo (com os mesmos dados da Figura 1) a vLL $_{\log -\log }$ é $10^{0,952}=8,95 \mathrm{~km} / \mathrm{h}$.

\section{Ajuste das curvas}

A qualidade do ajuste das curvas foi calculada conforme Hughson et al. (1987):

$$
\begin{aligned}
E Q M_{E X P}= & S Q R_{E X P} /(n-3) \text { e } \mathrm{EQM}_{\text {log-log }}= \\
& \mathrm{SQR}_{\text {log-log }} /(n-4)
\end{aligned}
$$

em que, EQM é o erro quadrático médio, SQR é a soma dos quadrados dos resíduos, EXP se refere ao modelo exponencial mais constante com três parâmetros independentes, log-log se refere ao modelo logarítmico com quatro parâmetros independentes, e $n$ equivale ao número da amostras de lactato-velocidade para cada sujeito.

As variáveis $S Q R$ e EQM foram determinadas nas coordenadas originais para ambos os modelos para que pudessem ser comparadas Hughson et al. (1987). Adicionalmente, o coeficiente de determinação $\left(R^{2}\right)$ foi calculado para os dois modelos.

\section{Análises estatísticas}

Os dados são apresentados como média \pm DP. A normalidade dos dados foi verificada através do teste de Shapiro-Wilk. Utilizou-se ANOVA para medidas repetidas e post hoc de Bonferroni na comparação entre os métodos. $O$ teste $\mathrm{t}$ de Student pareado foi utilizado na comparação entre as variáveis SQR, EQM e $R^{2}$. A correlação entre os métodos foi feita utilizando-se o coeficiente de correlação de Pearson. Para a análise de concordância entre os métodos foi utilizada a análise de Bland-Altman (BLAND; ALTMAN, 1986). As análises foram realizadas com o auxílio do Statistical Package for the Social Sciences versão 17.0 (SPSS Inc. USA) adotandose o nível de significância de $P<0,05$.

\section{Resultados}

Todas as participantes completaram pelo menos seis estágios no teste incremental em esteira ergométrica e atenderam a pelo menos dois critérios de máximo esforço. Duas participantes atenderam a quatro critérios, três participantes atenderam a apenas dois critérios e quinze participantes atenderam a três critérios. Entretanto, como os valores de uma participante apresentaram grande afastamento dos valores das demais participantes, influenciando bastante na análise de concordância entre os métodos, os valores desta participante foram identificados como outlier e eliminados das análises.

$\mathrm{Na}$ análise das 16 participantes restantes não houve diferença significante $(P>0,05)$ entre os ajustes exponencial mais constante (SQR: 1,01 \pm $1,23 \mathrm{mM}$; EQM: $0,21 \pm 0,24 \mathrm{mM}$; $\left.R^{2}: 0,95 \pm 0,06\right)$ e logarítmico (SQR: 0,88 $\pm 1,27 \mathrm{mM}$; EQM: 0,25 \pm 
$\left.0,32 \mathrm{mM} ; R^{2}: 0,95 \pm 0,05\right)$ aplicando-se o teste $t$ de Student pareado. A tabela 1 apresenta as variáveis fisiológicas do teste incremental. Não houve diferença significante $(P>0,05)$ entre as velocidades $v L L_{\text {log-log }}$ e $v L L_{D \max }$. No entanto, a
$\mathrm{vLL}_{3,5 \mathrm{~mm}}$ se mostrou significantemente superior à $v L_{\text {log-log }}(P<0,001)$ e à $v L L_{\text {Dmax }}(P=0,024)$, em que entre as 16 participantes a $v L_{3,5 \mathrm{mM}}$ foi superior à vLL $L_{\text {log-log }}$ para as 16 participantes $\mathrm{e}$ superior à $v L L_{D \max }$ para 13 participantes.

Tabela 1. Variáveis fisiológicas obtidas no teste incremental $(n=16)$

\begin{tabular}{|c|c|}
\hline Variáveis & Média \pm DP \\
\hline PSEpico (escala de Borg 6-20) & $18,9 \pm 1,4$ \\
\hline LApico (mM) & $7,3 \pm 2,3$ \\
\hline Vpico $(\mathrm{km} / \mathrm{h})$ & $13,4 \pm 1,0$ \\
\hline$v L_{\text {log-log }}(\mathrm{km} / \mathrm{h}) /(\%$ pico $)$ & $10,3 \pm 1,4^{*} / 76,9 \pm 9,5 \% *$ \\
\hline $\mathrm{vLL}_{\text {Dmax }}(\mathrm{km} / \mathrm{h}) /(\%$ pico $)$ & $10,9 \pm 0,8^{*} / 81,4 \pm 2,1 \% *$ \\
\hline $\mathrm{vLL}_{3,5 \mathrm{~mm}}(\mathrm{~km} / \mathrm{h}) /(\%$ Vpico $)$ & $11,6 \pm 1,2 / 86,4 \pm 7,7 \%$ \\
\hline
\end{tabular}

* $P<0,05$ em relação à vLL $3,5 \mathrm{mM}$.

Nota: PSEpico: percepção subjetiva de esforço ao final do teste incremental; LApico: concentração de lactato sanguíneo ao final do teste incremental; Vpico: velocidade atingida ao final do teste incremental; vLL: velocidade de corrida no limiar de lactato determinada pelos métodos log-log (vLL log-log $)$, Dmax (vLL $\left.L_{\text {Dmax }}\right)$ e concentração fixa em 3,5 mM (vLL $\left.3,5 \mathrm{~mm}\right)$.

A Figura 3 (esquerda) apresenta a regressão linear entre as velocidades $v L L_{\text {log-log }}$ e $v L L_{D \max }$. $O$ coeficiente de correlação de Pearson foi de $r=$ $0,65 \quad(P<0,01) \quad$ indicando uma correlação moderada entre os dois métodos. A correlação entre a $v L L_{3,5 \mathrm{mM}}$ e as velocidades $v L L_{\text {Dmax }}$ e $v L L_{\text {log- }}$ log foi estatisticamente significante de $r=0,70$ $(P<0,01)$ e $r=0,78(P<0,001)$, respectivamente. Verifica-se na figura 3 através da reta identidade (tracejada) que a $v L L_{\text {Dmax }}$ foi superior à $v L L_{\text {log-log }}$ para 10 participantes, e inferior para as seis restantes. Verifica-se também que poucos pontos situaram-se junto à reta de regressão, demonstrando um grau elevado de dispersão dos dados, e diminuindo o poder de explicação $\left(R^{2}=\right.$ $0,42)$ da variável vLL log-log pela variável vLL $L_{\text {Dmax. }} \mathrm{Na}$ figura 3 (direita) é apresentado o gráfico de BlandAltman entre as velocidades no LL determinadas pelos métodos Dmax e log-log. Como houve correlação estatisticamente significante entre os resíduos $\left(v L L_{D \max }-v L L_{\text {log-log }}\right)$ e a média das velocidades $(r=-0,63 ; P<0,01)$, o viés $\pm 1,96 \mathrm{DP}$ foi de $7,74-\left(0,67 \times\left(v L L_{D \max }+v L L_{\log -\log }\right) / 2\right) \pm 1,67$ $\mathrm{km} / \mathrm{h}$, determinados de acordo com Bland e Altman (1999).
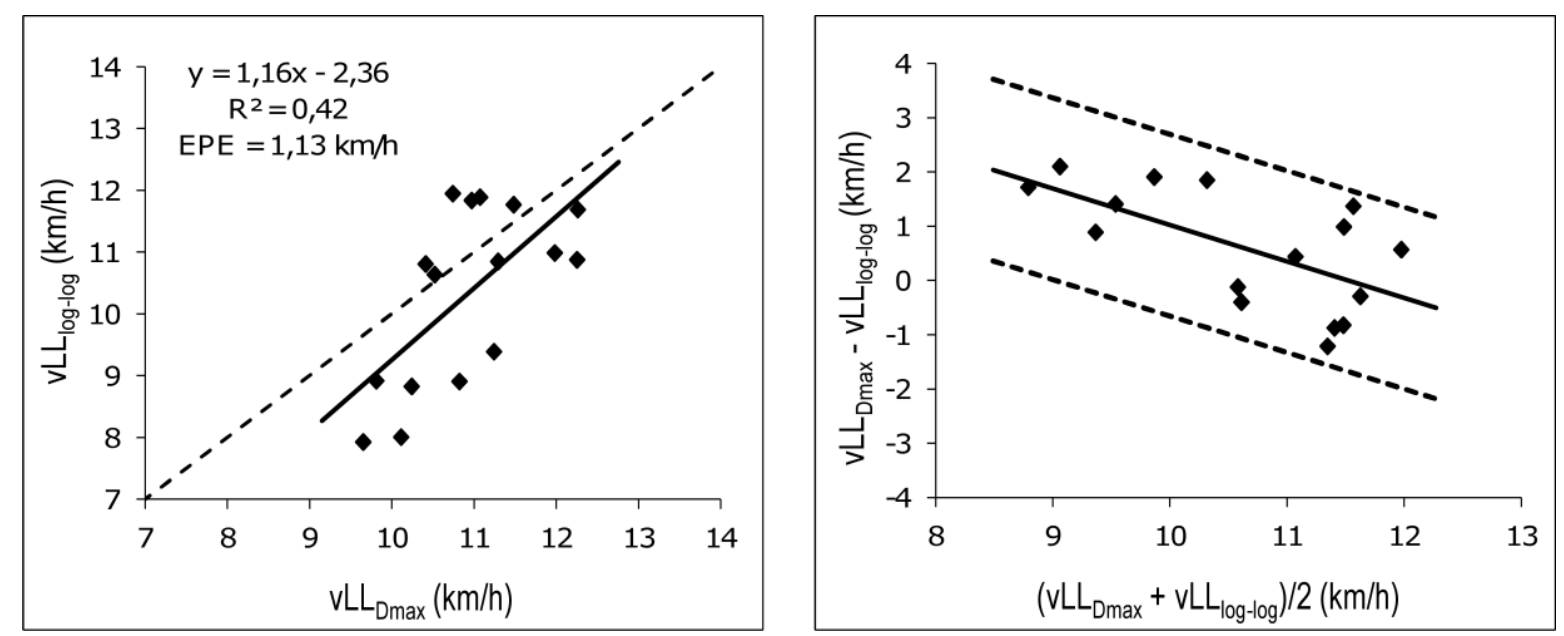

Figura 3. Regressão linear (esquerda) e gráfico de Bland-Altman (direita) entre as velocidades no limiar de lactato determinadas pelos métodos Dmax $\left(v L L_{D \max }\right)$ e log-log $\left(v L L_{\text {log-log }}\right)$. Á esquerda, a correlação foi $r=$ $0,65(P<0,01)$. À direita, o viés (linha contínua) $\pm 1,96 \mathrm{DP}$ (linhas tracejadas) foi de $7,74-\left(0,67 \times\left(\mathrm{vLL} \mathrm{Dmax}_{\max }+\right.\right.$ $\left.\left.\mathrm{vLL}_{\log -\log }\right) / 2\right) \pm 1,67 \mathrm{~km} / \mathrm{h}(n=16)$. 
A Figura 4 apresenta $\circ$ gráfico Bland-Altman entre a $v L_{3,5 m M}$ e a $v L L_{\text {Dmax }}$ (esquerda) e entre a $v L L_{3,5 \mathrm{~mm}}$ e a $v L L_{\text {log-log }}$ (direita). Na figura 4 , à esquerda, observa-se que houve correlação estatisticamente significante entre os resíduos $\left(\mathrm{vLL}, 5 \mathrm{~mm}-\mathrm{vLL} L_{D \max }\right)$ e a média dessas velocidades $(r=0,51 ; P<0,05)$, em que o viés $\pm 1,96 \mathrm{DP}$ foi de $-4,75+\left(0,48 \times\left(v L L_{3,5 \mathrm{mM}}+v L L_{\text {Dmax }}\right) / 2\right) \pm 1,48$ $\mathrm{km} / \mathrm{h}$. Na figura 4 , à direita, a correlação não foi estatisticamente significante $(P>0,05)$, com viés \pm
1,96 DP de $1,27 \pm 1,77 \mathrm{~km} / \mathrm{h}$. Como as escalas dos eixos da figura 3 (direita) e 4 (direita e esquerda) são iguais, verifica-se visualmente que a amplitude entre os limites de concordância inferior e superior nos três gráficos são semelhantes, variando entre $\pm 1,48$ e $\pm 1,77 \mathrm{~km} / \mathrm{h}$, e desta forma a concordância estatística entre os métodos foi semelhante, apesar de a $\mathrm{vLL}_{3,5 \mathrm{mM}}$ superestimar tanto a $v L L_{\text {log-log }}$ como a $v L L_{\text {Dmax }}$.
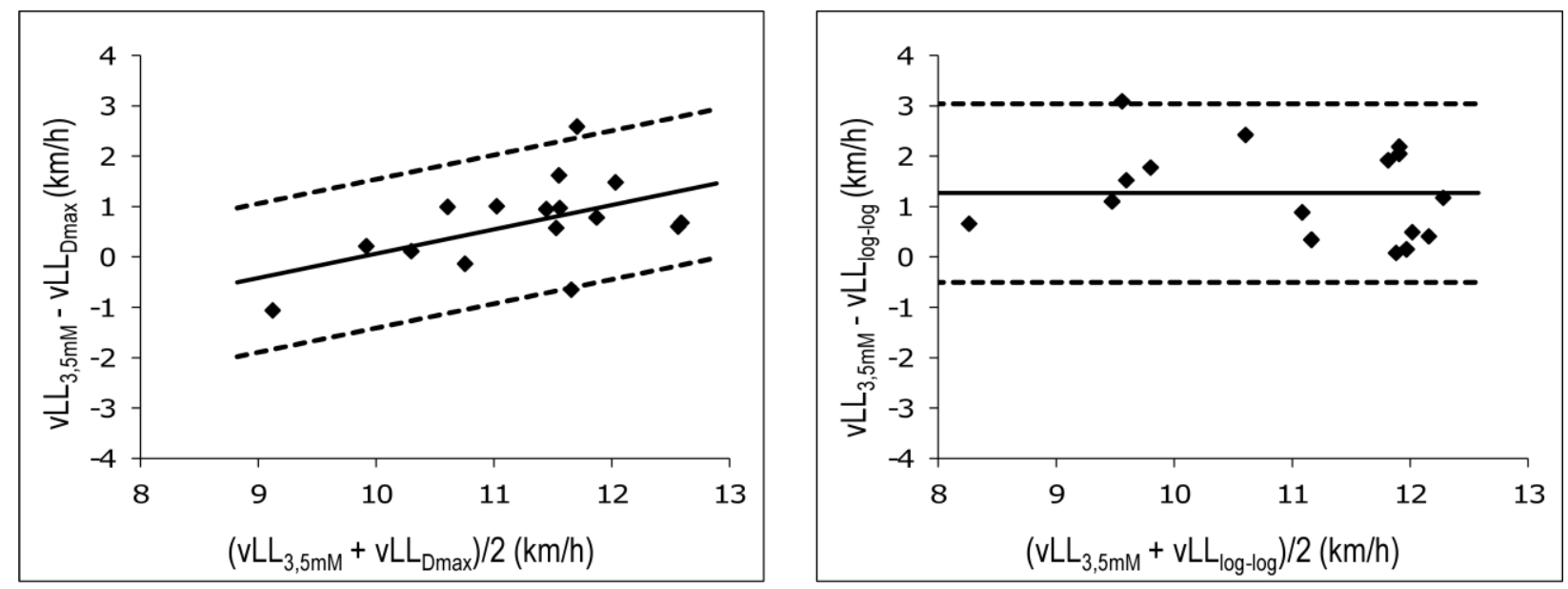

Figura 4. Gráfico Bland-Altman: análise de concordância entre as velocidades de corrida no limiar de lactato determinadas pelos métodos da concentração fixa de lactato em 3,5 $\mathrm{mM}\left(\mathrm{vLL} \mathrm{L}_{3,5 \mathrm{mM}}\right)$ e Dmax ( $\mathrm{vL} \mathrm{L}_{\mathrm{Dmax})}$

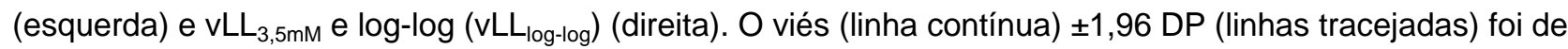
$-4,75+\left(0,48 \times\left(v L L_{3,5 \mathrm{mM}}+\mathrm{vLL}_{\mathrm{Dmax}}\right) / 2\right) \pm 1,48 \mathrm{~km} / \mathrm{h}$ (esquerda) e 1,27 $\pm 1,77 \mathrm{~km} / \mathrm{h}$ (direita) $(n=16)$.

\section{Discussão}

Este estudo examinou se os métodos automáticos e individualizados log-log e Dmax detectam o LL em intensidades semelhantes, de modo que permitam a aplicação destes métodos de maneira intercambiável. Também foi examinado se a relação entre eles é melhor do que a relação deles com 0 método não individualizado de detecção do $\mathrm{LL}$ na concentração fixa de lactato sanguíneo em 3,5 $\mathrm{mM}$. O principal achado foi que os métodos loglog e Dmax não apresentaram um maior nível de correlação e concordância entre eles do que quando comparados com 0 método de concentração fixa de lactato sanguíneo em 3,5 $\mathrm{mM}$. Desta forma, a utilização destes métodos de forma intercambiável deve ser feita com cautela, visto terem sido observadas diferenças de até $15 \%$ entre eles.

Uma vez que o LL é utilizado para predizer o desempenho, prescrever a intensidade de treinamento e também avaliar os efeitos do treinamento, sua determinação se tornou muito importante especialmente para técnicos e atletas. Entretanto, como ainda não existe na literatura nenhum método que seja considerado "padrão ouro" para a determinação do LL, a comparação entre métodos se torna importante, verificando-se as correlações e concordâncias entre eles, a fim de saber se são produzidas estimativas semelhantes do LL.

Para essa comparação, precisamos primeiramente garantir que os métodos foram aplicados adequadamente. Um dos requisitos para o método Dmax é que o teste tenha sido máximo. Isso foi garantido verificando que todas as participantes atenderam a pelo menos dois critérios de esforço máximo, sendo que a maioria atendeu a três critérios. Outro ponto importante é a curva utilizada para o ajuste dos dados. Nicholson e Sleivert (2001) utilizaram a equação exponencial mais constante para 0 ajuste dos dados e verificaram que a vLL $L_{D \max }$ apresentou uma alta correlação com a velocidade de corrida de $10 \mathrm{~km}(r=0,86 ; P<0,001)$. Por outro lado,

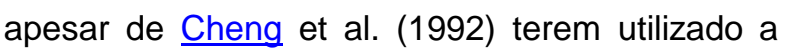


curva polinomial de terceira ordem no desenvolvimento do método Dmax, não existe nenhum estudo na literatura que tenha demonstrado que esta função representa o aumento na concentração de lactato em função do aumento da intensidade de exercício. Já Hughson et al. (1987) demonstraram que a curva exponencial mais constante pode representar a resposta fisiológica do lactato durante um teste progressivo, visto que esta se ajustou muito bem à curva lactato-intensidade. Diante disso, optamos por utilizar o ajuste exponencial mais constante, o qual se ajustou bem aos dados $\left(R^{2}=0,94\right)$, tal como o ajuste logarítmico $\left(R^{2}=0,94\right)$, sem que houvesse diferença significante entre estes dois ajustes.

As participantes completaram entre seis e noves estágios do teste incremental e optamos por utilizar a relação lactato-velocidade para a determinação da vLL. O impacto da quantidade de dados para o ajuste das curvas e o impacto da utilização da relação lactato-velocidade e não da relação lactato- $\mathrm{VO}_{2}$ não foi verificado pelo fato deste estudo não ter sido delineado para responder a essas questões. No entanto, entendemos que com essa quantidade mínima de estágios completados pelas participantes já tenha sido possível atender o escopo deste estudo. Além disso, a relação lactato-velocidade, bem como o ajuste exponencial, já foram validados por Nicholson e Sleivert (2001).

Conforme tabela 1, não houve diferença significativa entre as velocidades de corrida no limiar de lactato determinadas pelos métodos Dmax e log-log $(P>0,05)$. Também não houve tendência de um método subestimar ou superestimar o outro. Em contraste, a $v L_{3,5 \mathrm{mM}}$ apresentou a tendência de superestimar tanto a $v L L_{\text {log-log }}$ como a vLL Dmax. Considerando que os métodos Dmax e log-log são métodos individualizados de detecção automática do LL era de se esperar que ambos apresentassem uma forte correlação, a qual não ocorreu. Ainda que fosse encontrada uma alta correlação, isto não seria garantia de um elevado nível de concordância, e vice-versa, conforme se infere dos estudos de Davis et al. (2007) e Thomas et al. (2008).

Davis et al. (2007) compararam três métodos de detecção do LL durante um teste progressivo em ciclo ergômetro, não encontrando diferença significativa entre os métodos de detecção visual
$(104,5 \mathrm{~W}), \log -\log (105,1 \mathrm{~W})$ e intensidade de exercício que antecede a um aumento na concentração de lactato maior que 0,5 mM $\left(L_{0,5 \mathrm{~mm}}\right) \quad(103,2 \mathrm{~W})$. Os três métodos foram altamente correlacionados $(0,977 \leq r \leq 0,992)$, entretanto, o viés (diferença entre as médias) entre os métodos log-log e $\mathrm{LL}_{0,5 \mathrm{mM}}$, por exemplo, foi de 1,9 W com limites de concordância ( $\pm 2 \mathrm{DP}$ ) entre $-18,6 \mathrm{~W}$ e $+22,5 \mathrm{~W}$, podendo levar a uma diferença no LL maior que $20 \%$ para um mesmo indivíduo.

Thomas et al. (2008) também compararam alguns índices de lactato obtidos de testes realizados em ciclo ergômetro e não encontraram diferença significante $(P>0,05)$, por exemplo, entre o método da concentração fixa em $4 \mathrm{mM}$ $(158,8 \mathrm{~W})$ e o método $\operatorname{Dmax}(171,0 \mathrm{~W})$, havendo uma alta correlação entre ambos $(r=0,84 ; P<$ $0,05)$. O viés foi de 12,2 com limites de concordância ( \pm 2 DP) entre $-16,9$ e +41,3 W. Assim, diferenças maiores que $20 \%$ também podem ser encontradas entre estes dois LL para um mesmo indivíduo.

Desta forma, é necessária uma análise da concordância entre os métodos tal como proposta por Bland e Altman (1986; 1999) para que se verifique 0 viés e os limites de concordância. Verifica-se através da figura 3 , à esquerda, que os dados ficaram bem dispersos ao redor da reta de regressão. Além disso, o poder de explicação da variável dependente ( $v L L_{\text {log-log }}$ ) através da variável independente ( $v L L_{\text {Dmax }}$ ) foi baixo. Aliada a esta informação, na figura 3 à direita, o gráfico de Bland-Altman apresenta os limites de concordância entre os métodos Dmax e log-log. 0 viés entre os dois métodos pode ser considerado pequeno e não representa nenhum problema, visto que pode ser corrigido utilizando-se a equação de regressão linear apresentada na figura 3 à esquerda. O principal problema é a variabilidade representada pelos limites de concordância de $\pm 1,67 \mathrm{~km} / \mathrm{h}$. Por exemplo, para um indivíduo com uma vLL média de $10 \mathrm{~km} / \mathrm{h}$, o viés seria de $1,04 \mathrm{~km} / \mathrm{h}$ com os limites de concordância entre $-0,63$ e $2,71 \mathrm{~km} / \mathrm{h}$. Esta diferença, da ordem de $\pm 15 \%$, pode ser considera moderada dependendo da sua aplicação por atletas, técnicos e pesquisadores, mas não pode ser desconsiderada.

Em relação à comparação dos métodos individualizados com o método não individualizado, verifica-se que apesar da $v L L_{3,5 \mathrm{mM}}$ 
tender a superestimar a $v L L_{\text {log-log }}$ e a $v L L_{D \max }$, a variabilidade entre aquela e estas foi da mesma ordem que a variabilidade entre a $v L^{2} L_{\log -\log } \mathrm{e} a$ vLLDmax.

Diante disso, a principal conclusão deste estudo é que apesar dos métodos Dmax e log-log apresentarem as características em comum de serem métodos objetivos que detectam matematicamente o LL, levando em consideração as respostas metabólicas de cada indivíduo, estes métodos não apresentaram um maior nível de correlação e concordância entre si do que quando comparados com o método não individualizado de detecção do LL na concentração fixa de lactato sanguíneo em 3,5 mM. Entre as limitações deste estudo inclui-se tanto o tamanho amostral como a ausência de participantes do gênero masculino na obtenção dos resultados. Ainda assim, a utilização destes métodos de forma intercambiável deve ser feita com cautela por técnicos, atletas e pesquisadores devido ao fato de terem sido observadas diferenças de até aproximadamente $15 \%$ entre eles.

\section{Referências}

BEAVER, W. L.; WASSERMAN, K.; WHIPP, B. J. Improved detection of lactate threshold during exercise using a log-log transformation. Journal of Applied Physiology, Bethesda, v. 59, n. 6, p. 1936-1940, 1985. Disponível em:

$<$ http://jap.physiology.org/cgi/content/abstract/59/6 /1936>. Acesso em: 2 mar. 2012.

BILLAT, V. L. Use of blood lactate measurements for prediction of exercise performance and for control of training. Sports Medicine, Auckland, v. 22, n. 3, p.157-175, 1996. Disponível em: $<$ http://adisonline.com/sportsmedicine/Abstract/19 96/22030/Use of Blood Lactate Measurements for Prediction.3.aspx>. Acesso em: 2 mar. 2012.

BISHOP, D.; JENKINS, D. G.; MACKINNON, L. T. The relationship between plasma lactate parameters, Wpeak and 1-h cycling performance in women. Medicine and Science in Sports and Exercise, Philadelphia, v. 30, n. 8, p. 1270-1275, 1998. Disponível em:

$<$ http://journals.lww.com/acsm-

msse/Abstract/1998/08000/The relationship betw een plasma lactate.14.aspx>. Acesso em: 2 mar. 2012.

BLAND, J. M.; ALTMAN, D. G. Statistical methods for assessing agreement between two methods of clinical measurement. Lancet, London, v. 327, n. 8476, p. 307-310, 1986. Disponível em: $<$ http://dx.doi.org/10.1016/S0140-6736(86)908378ㄱ. Acesso em: 2 mar. 2012.
BLAND, J. M.; ALTMAN, D. G. Measuring agreement in method comparison studies. Statistical Methods in Medical Research, London, v. 8, n. 2, p.135-160, 1999. Disponível em:

$<$ http://smm.sagepub.com/content/8/2/135.abstrac t>. Acesso em: 2 mar. 2012.

BORG, G. A. Psychophysical bases of perceived exertion. Medicine and Science in Sports and Exercise, Philadelphia, v. 14, n. 5, p. 377-381, 1982. Disponível em: $<$ http://journals.lww.com/acsmmsse/Abstract/1982/05000/Psychophysical bases of perceived exertion.12.aspx >. Acesso em: 2 mar. 2012.

CHENG, B.; KUIPERS, H.; SNYDER, A. C.; KEIZER, H. A.; JEUKENDRUP, A.; HESSELINK, $M$. A new approach for the determination of ventilatory and lactate thresholds. International Journal of Sports Medicine, Stuttgart, v. 13, n. 7, p. 518-522, 1992. Disponível em: <http://dx.doi.org/10.1055/s-2007-1021309>. Acesso em: 2 mar. 2012.

COYLE, E. F.; MARTIN, W. H.; EHSANI, A. A.; HAGBERG, J. M.; BLOOMFIELD, S. A.; SINACORE, D. R.; HOLLOSZY J. O. Blood lactate threshold in some well-trained ischemic heart disease patients. Journal of Applied

Physiology, Bethesda, v. 54, n. 1, p. 18-23, 1983. Disponível em:

$<$ http://jap.physiology.org/cgi/content/abstract/54/1 /18>. Acesso em: 2 mar. 2012.

DAVIS, J. A.; ROZENEK, R.; DECICCO, D. M.; CARIZZI, M. T.; PHAM, P. H. Comparison of three methods for detection of the lactate threshold. Clinical Physiology and Functional Imaging, Oxford, v. 27, n. 6, p. 381-384, 2007. Disponível em: <http://dx.doi.org/10.1111/i.1475097X.2007.00762.x>. Acesso em: 2 mar. 2012.

FARRELL, P. A.; WILMORE, J. H.; COYLE, E. F.; BILLING, J. E.; COSTILL, D. L. Plasma lactate accumulation and distance running performance. Medicine and Science in Sports, Philadelphia, v. 11, n. 4, p. 338-344, 1979. Disponível em: $<$ http://journals.lww.com/acsmmsse/Abstract/1979/01140/Plasma lactate accu mulation and distance running.5.aspx >. Acesso em: 2 mar. 2012.

HECK, H.; MADER, A.; HESS, G.; MUCKE, S.; MULLER, R.; HOLLMANN, W. Justification of the 4-mmol// lactate threshold. International Journal of Sports Medicine, Stuttgart, v. 6, n. 3, p. 117130, 1985. Disponível em: <http://dx.doi.org/10.1055/s-2008-1025824>. Acesso em: 2 mar. 2012. 
HUGHSON, R. L.; WEISIGER, K. H.; SWANSON, G. D. Blood lactate concentration increases as a continuous function in progressive exercise.

Journal of Applied Physiology, Bethesda, v. 62, n. 4, p. 1975-1981, 1987. Disponível em:

$<$ http://iap.physiology.org/cgi/content/abstract/62/5 /1975>. Acesso em: 2 mar. 2012.

KINDERMANN, W.; SIMON, G.; KEUL, J. The significance of the aerobic-anaerobic transition for the determination of work load intensities during endurance training. European Journal of Applied Physiology and Occupational Physiology, Heidelberg, v. 42, n. 1, p. 25-34, 1979. Disponível em: <http://dx.doi.org/10.1007/BF00421101>. Acesso em: 2 mar. 2012.

MYERS, J.; WALSH, D.; BUCHANAN, N.; MCAULEY, P.; BOWES, E.; FROELICHER, V. Increase in blood lactate during ramp exercise: comparison of continuous and threshold models. Medicine and Science in Sports and Exercise, Philadelphia, v. 26, n. 11, p. 1413-1419, 1994. Disponível em: <http://journals.lww.com/acsmmsse/Abstract/1994/11000/Increase in blood lac tate during ramp exercise .18.aspx>. Acesso em: 2 mar. 2012.

NICHOLSON, R. M.; SLEIVERT, G. G. Indices of lactate threshold and their relationship with $10-\mathrm{km}$ running velocity. Medicine and Science in

Sports and Exercise, Philadelphia, v. 33, n. 2, p. 339-342, 2001. Disponível em:

$<$ http://journals.lww.com/acsmmsse/Abstract/2001/02000/Indices of lactate thr eshold and their.26.aspx>. Acesso em: 2 mar. 2012.

PAPADOPOULOS, C.; DOYLE, J. A.; LABUDDE, B. D. Relationship between running velocity of 2 distances and various lactate parameters. International Journal of Sports Physiology and Performance, Champaign, v. 1, n. 3, p. 270-283, 2006. Disponível em:

$<$ http://journals. humankinetics.com/ijspp-backissues/lJSPPVolume1/ssue3September/Relations hipBetweenRunningVelocityof2DistancesandVario usLactateParameters>. Acesso em: 2 mar. 2012.

SJODIN, B.; JACOBS, I. Onset of blood lactate accumulation and marathon running performance. International Journal of Sports Medicine, Stuttgart, v. 2, n. 1, p. 23-26, 1981. Disponível em: <http://dx.doi.org/10.1055/s-2008-1034579>. Acesso em: 2 mar. 2012.

STRATTON, E.; O'BRIEN, B. J.; HARVEY, J.; BLITVICH, J.; MCNICOL, A. J.; JANISSEN, D.; PATON, C.; KNEZ, W. Treadmill velocity best predicts 5000-m run performance. International Journal of Sports Medicine, Stuttgart, v. 30, n. 1, p. 40-45, 2009. Disponível em:
$<$ http://dx.doi.org/10.1055/s-2008-1038761>. Acesso em: 2 mar. 2012.

THOMAS, V.; COSTES, F.; CHATAGNON, M.; POUILLY, J. P.; BUSSO, T. A comparison of lactate indices during ramp exercise using modeling techniques and conventional methods. Journal of Sports Sciences, Philadelphia, v. 26, n. 13, p. 1387-1395, 2008. Disponível em: <http://dx.doi.org/10.1080/02640410802104920>. Acesso em: 2 mar. 2012.

TOKMAKIDIS, S. P.; LÉGER, L. A.; PILIANIDIS, $T$. C. Failure to obtain a unique threshold on the blood lactate concentration curve during exercise. European Journal of Applied Physiology and Occupational Physiology, Heidelberg, v. 77, n. 4, p. 333-342, 1998. Disponível em: $<$ http://dx.doi.org/10.1007/s004210050342>. Acesso em: 2 mar. 2012.

YOSHIDA, T.; CHIDA, M.; ICHIOKA, M.; SUDA, Y. Blood lactate parameters related to aerobic capacity and endurance performance. European Journal of Applied Physiology and Occupational Physiology, Heidelberg, v. 56, n. 1, p. 7-11, 1987. Disponível em: $<$ http://dx.doi.org/10.1007/BF00696368>. Acesso em: 2 mar. 2012.

\section{Endereço:}

Fabiana Andrade Machado

Departamento de Educação Física - Universidade Estadual de Maringá

Av. Colombo, 5.790 - Jd. Universitário

Maringá PR Brasil

87020-900

Telefone: (44) 3011-4315

e-mail: famachado@uem.br

Recebido em: 6 de outubro de 2010.

Aceito em: 1 de fevereiro de 2012.

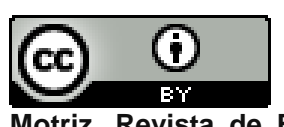

Motriz. Revista de Educação Física. UNESP, Rio Claro, SP, Brasil - elSSN: 1980-6574 - está licenciada sob Creative Commons - Atribuição 3.0 